\title{
Robotic INNER Signal Propagation AND RANDOM ACCESS OVER HYBRID ACCESS SCHEME
}

\author{
Abir Hossain, Zhenni Pan, Megumi Saito, Jiang Liu and Shigeru Shimamoto \\ Department of Computer Science and Communications Engineering, \\ Waseda University, Tokyo 169-0072, Japan
}

\begin{abstract}
This paper proposes a Hybrid Access Scheme (HAS) aiming to convert a future robot's backend communication system by a finite number of sensors instead of using a lot of wires. To replace this communication, the HAS needs to assure higher reliability within stringent low latency packet transmission. In this paper, the HAS utilizes the packet diversity principle and forward multiple copies of the same packet over the massive number of subcarrier channels. The HAS assigns the random accessing to select a subcarrier channel for general packet transmitting sensors. The audio and video sensors transmit packets over the dedicated channels to avoid collisions. The HAS system allows transmitting audio, video and general sensors simultaneously. The minimum number of subcarriers to satisfy the URLLC reliability requirement of $99.999 \%$ is evaluated for different packet duplications over different arrival condition.The HAS system's reliability and collision probability are evaluated in MATLAB simulator for different packet duplication over different arrival condition. Moreover, the signal propagation expressions are captured using ANSYS HFSS software for rectangular and circular transmission medium over the $900 \mathrm{MHz}, 2.4 \mathrm{GHz}, 24 \mathrm{GHz}$, and $55 \mathrm{GHz}$ frequency bands for different structural configurations.
\end{abstract}

\section{KEYWORDS}

Hybrid Access Scheme, Signal Propagation, Random Access, reliability, URLLC

\section{INTRODUCTION}

In the state-of-the-art technology, wireless sensor converted system is getting more attention day by day. A wireless sensor converted system being demanded due to its mobility, scalability, flexibility, cost-efficiency, and faster deployment. However, a robot consists of multiple elements, and many wires are usually required as the communication backbone among attached different elements [1]. A wire connected robot gain more weight and consume more power for its usual movement. Moreover, the maintenance become complex when any internal wires get disconnected during operation. By increasing the demands of wireless sensor connected system and to resolve the defined problems, we intend to convert a wire system into a wireless one by a Hybrid Access Scheme (HAS). A typical wireless sensor connected robot is presented in Figure 1. As illustrated in Figure 1, there are three types of sensors (Audio, video and general sensor) deployed ubiquity over the robotic structure and utilize hybrid access scheme to ensure ultra reliable low latency communication. The $3 \mathrm{GPP}$ sets the standard for Ultra-Reliable Low Latency Communication (URLLC) reliability requirement for a single packet transmission having size of 32 bytes is $1-10^{-5}$ (alternatively $99.999 \%$ ) with an interface latency of $1 \mathrm{~ms}[2,3]$. Due to ensure this higher reliability and latency, the HAS adopts packet diversity principle and transmits over massive number of subcarrier channels in almost error-free and interference-free manner. 


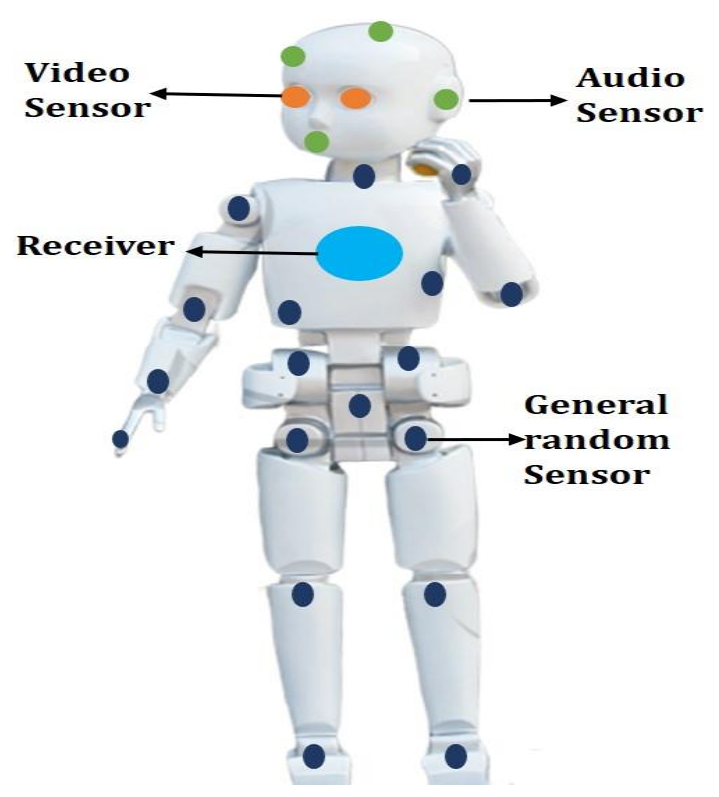

Figure 1. Wireless sensor connected robot.

The HAS system assumed to assign a massive number of orthogonal subcarrier channels to transmit packets from sensor to receiver site. Due to consider small area inside a robotic structure, we assumed that the assign massive number of channels will not interfere with the central mobile bandwidth assignment.

\subsection{Related works}

A several number of research works conducted to convert an established into a wireless system by a finite number of sensors. Under the $5 \mathrm{G}$ communication domain, a sensor communication based structural health monitoring and early earthquake warning system is presented in [4] that give a proposal of monitoring and warning generation system in presence of seismic events. A wireless sensor connected structural health monitoring system proposed for an airplane is studied in [5] where sensor nodes observing the health conditions of an airplane deployed and covered the entire area of an aircraft. These nodes evaluate throughput, the data dropped rate and the delay for a maximum of 7 nodes as approximately $12000 \mathrm{bps}, 180 \mathrm{bps}$, and115ms, respectively. A real time elderly health care monitoring system is proposed in[6] that utilize a wireless sensor network where bracelet-type devices are equipped with sensors applied to each patient under the monitoring system of the healthcare that received data in real time and stored in a central monitoring server. A spacecraft automation and communication based on sensor is studied in [7], that transmit $988 \mathrm{~B}$ payload of sensor data in UWB in spacecraft networks over the different frequency bands using a TDMA protocol having a short 5ms time slot. A wireless sensorconnected robot's OFSMA MAC protocol is accepted in [8], which considered only a single frequency band. The main objectives of that proposed work are to determine the minimum number of channels to satisfy $99.999 \%$ reliability and air-interface latency for single-packet uplink communication.

The URLLC is a challenging service launched by $5 \mathrm{G}$ communication due to its higher reliability requirement and lower latency bound. The URLLC is specially designed to overcome the mission-critical system $[9,10]$ where the link reliability is highly expected in a strict latency requirement. The URLLC service is highly demanded and attracts attention a lot of researcher 
International Journal of Computer Networks \& Communications (IJCNC) Vol.12, No.4, July 2020

due to its exciting and interesting applications. Among the different applications, the URLLC is applicable in reliable remote action with robots and coordination among vehicles [11], tactile internet [12], augmented or virtual reality (AR/VR) [13] and many more. This research work [14] presents an emerging system of URLLC and eMBB where the time is divided into slots and the time slot is further subdivided into minislots. The URLLC data is overlapped with eMBB traffic and transmitted over the channel using superposition/puncturing and received successfully using successive interference cancellation method. The URLLC is sensitive to its latency of the packet transmission. This research [15] covers URLLC and eMBB resource slicing over minislots in a risk-sensitive approach considering Conditional Value at Risk (CVaR) by managing properly both eMBB users scheduling and URLLC slot placement for the downlink communication. An unmanned aerial vehicle (UAV) based relay system is analyzed in [16] where the data is transmitted under the URLLC requirements. This research work subjected to optimize the blocklength allocation and minimize the decoding error probability under the latency requirement.

There are several random accessing protocols studied in the slotted ALOHA system to achieve the expected reliability and latency defined in URLLC. A contention-based slotted ALOHA system has been proposed in [17] that adopts multichannel, multipacket diversity and transmit multiple copies of the same packets in consecutive slots to improve system reliability, and to reduce latency to satisfy URLLC's conditions. Multichannel random accessing using OFDMA is analyzed in [18], which proposed a fast retrial algorithm to forward a packet into a frequency diversity system after a collision in a slotted ALOHA system. A multi-packet messaging system in a cluster-based environment analysed in [19] where the multiple packets forwarded into different slots and having an independent frequency is to improve the system throughput.

\subsection{Contributions}

The existing research work is not suitable to convert our proposed objective to convert a future robot's internal communication developed by sensors. To implement our proposed aim, we propose a hybrid access scheme. The main contributions of the paper are listed below:

- We propose a new hybrid access scheme (HAS) for a future robot's internal communication system. The HAS scheme ensures the higher packet transmission reliability of the audio, video, and randomly transmitted general sensors over the differently allocated subcarrier channels.

- We introduce a system model to represent the hybrid access scheme's total transmitted packet signal for a single Transmission Time Interval (TTI) slot, introduced AWGN noise into the channel and finally the received packet signal at the receiver site.

- We determine the minimum number of subcarriers required to satisfy the reliability of 99.999\% defined by URLLC for different packet duplication over variable arrival conditions.

- We evaluate the HAS system's packet transmission reliability and collision probability for fixed channel conditions by assigning sensor and subcarrier channel ratio as 1:1 with the variation of different packet duplications and low to higher arrival conditions. 
International Journal of Computer Networks \& Communications (IJCNC) Vol.12, No.4, July 2020

- We capture the signal propagation expression for rectangular and circular transmission medium as part of a robotic body structure for different structural configurations over diverse frequency bands and determine the success or failure transmission for various structural configurations.

The rest of this paper is organized as follows: Section 2 explains the details of the hybrid access scheme. In Section 3 represents the system model of the hybrid access scheme, presents the signal expression of the transmitted and received signals. Moreover, in section 3 presents a detailed explanation about the structure and properties of the rectangular and circular transmission medium. The simulation results are presented in Section 4. Finally, this paper is summarized in Section 5.

\section{HYBRID ACCESS SCHEME}

A Hybrid Access Scheme (HAS) is a combination of the Orthogonal Frequency Subcarrier-based Multiple Access Scheme (OFSMA) [7] and Dedicated Channel Access (DCA) presented in Figure 2. The general sensors transmit signal based on the OFSMA access scheme over the randomly selected subcarriers and the audio and video sensors transmit over the dedicated subcarriers assigned for transmission. The OFSMA access scheme presented in Figure 3. The OFSMA access scheme adopts a packet diversity principle and transmits multiple copies of the same packet over a different numbers of subcarrier channels in order to improve the reliability of the transmission. The system assumed that, there is no internal or self-packet collision among the transmitted duplicated packets emitted from a sensor for a single specific TTI. According to the OFSMA scheme, first a packet equivalent bit is created and copied for the number of duplicated packets. Each duplicated packet bits select an independent orthogonal subcarrier frequency randomly and perform modulation and mapping for transmission. After performing Inverse First Fourier Transform (IFFT), the signal of the packet bits is transmitted over Additive White Gaussian Noise (AWGN) channels to receiver. Due to multiple sensors transmission, there is a probability of collision, but it is minimized by introducing a massive number of subcarrier channels. Among the multiple transmission of a single packet, if at least one packet is successfully received by the receiver then it marked as a success and receiver ignores the other copies of the same duplicated packets. At the receiver side, uses a band pass filter to detect and collect the signal frequency. The receiver then started performing the reverse operation and decoded the signal bits from independent orthogonal subcarrier frequency and retrieve the original bit sequence. The audio and video sensors transmit packet over the dedicated channel. Due to the exclusive assignment of the channel, there is no probability of the collision, but a minor number of packets might be lost due to having low signal power at the receiver end. 


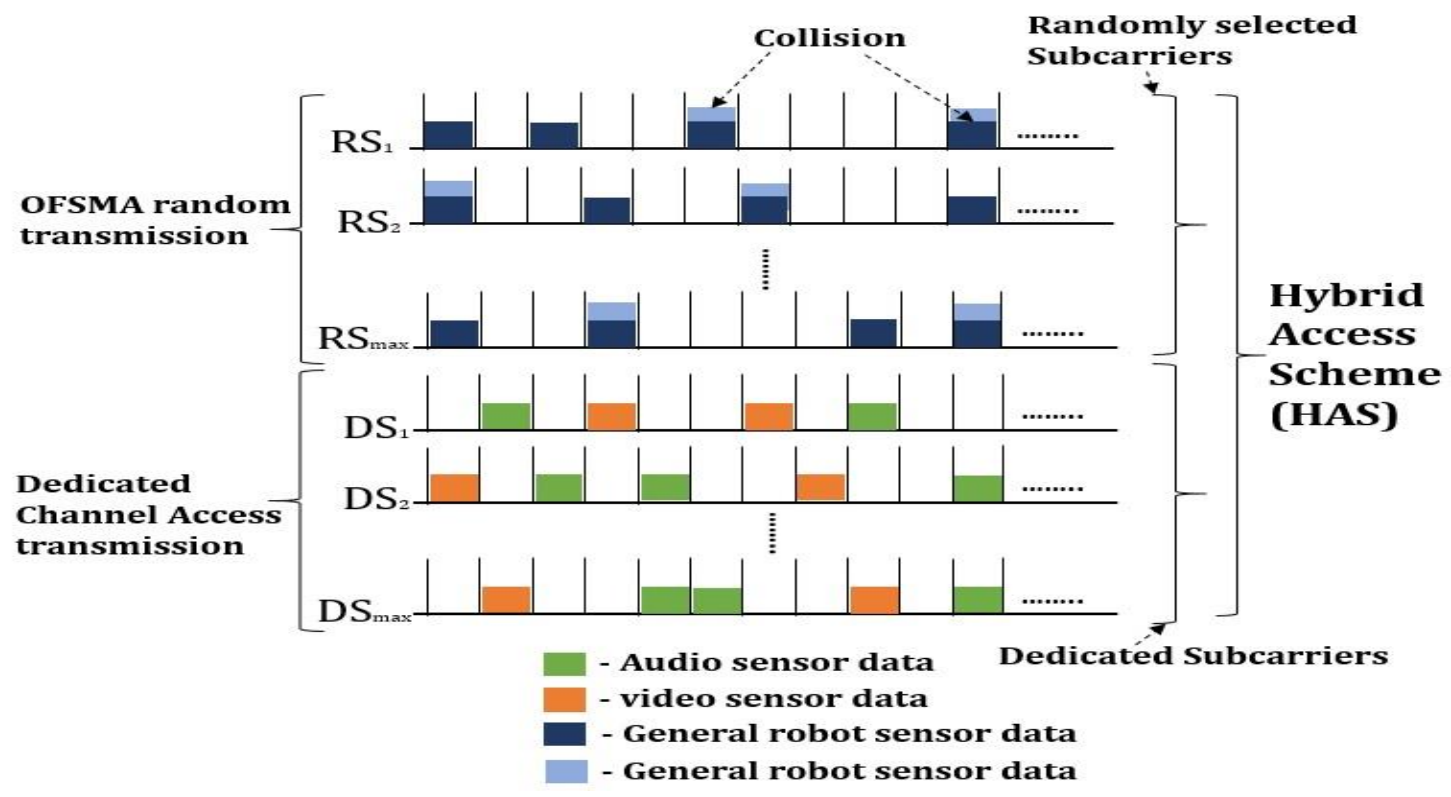

Figure 2. Hybrid Access Scheme

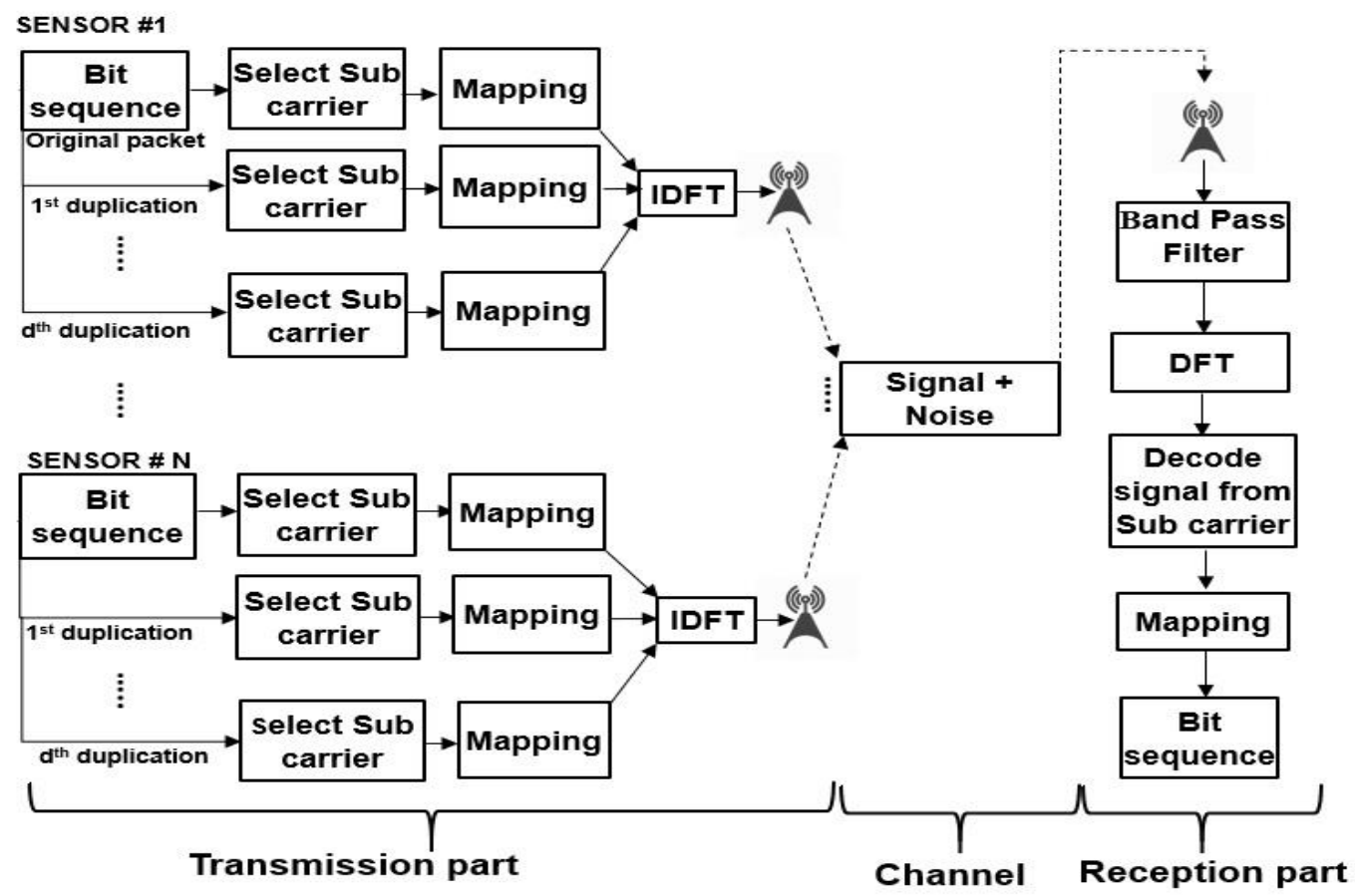

Figure 3. OFSMA Access Scheme

\section{SYSTEM MODEL}

We consider signal transmission and propagation in our system model. The first part of our system models presents the $H A S$ system design. The system design covers in a short range of the signal over the randomly selected subcarrier's transmitted packets for a single TTI, dedicated channels signal for a single TTI, considers AWGN noisy channels and finally the total received signal from all sensors in a single TTI. The second part of the system considers different 
International Journal of Computer Networks \& Communications (IJCNC) Vol.12, No.4, July 2020

structures as part of a robotic structure and captures the signal propagation expression for different frequency bands. The $H A S$ system design and robotic signal propagation explain below.

\subsection{HAS System Design}

An uplink transmission system is considered in our system present as shown in Figure 4. A finite number of sensors ubiquitously plotted around the centralized receiver. In our system, three types of sensors are considered as a) General sensor, b) Audio sensor and, c) Video sensor. The sensors transmit signal over the finite number of subcarrier channels. The subcarrier channels are also divided into two categories as random accessible channels and dedicated channels. The general sensors transmit signal data over the randomly selected channel and there might be a probability of a collision. To avoid the collision over the transmission, the system adopts the packet diversity principle. According to the packet diversity principle, each general sensor transmits multiple copies of the same packet over the randomly selected subcarrier channels. Among the multiple copies of the packet, if at least one packet is successfully received by the receiver, then the transmission is determined as a successful transmission. The system assigned a massive number of subcarrier channels in order to lower the probability of the collision. The general sensors are responsible for transmitting regular small payload data for communication. The dedicated subcarrier channels are allocated for audio and video sensors. There is no probability of collision in the dedicated channels for audio and video sensors.

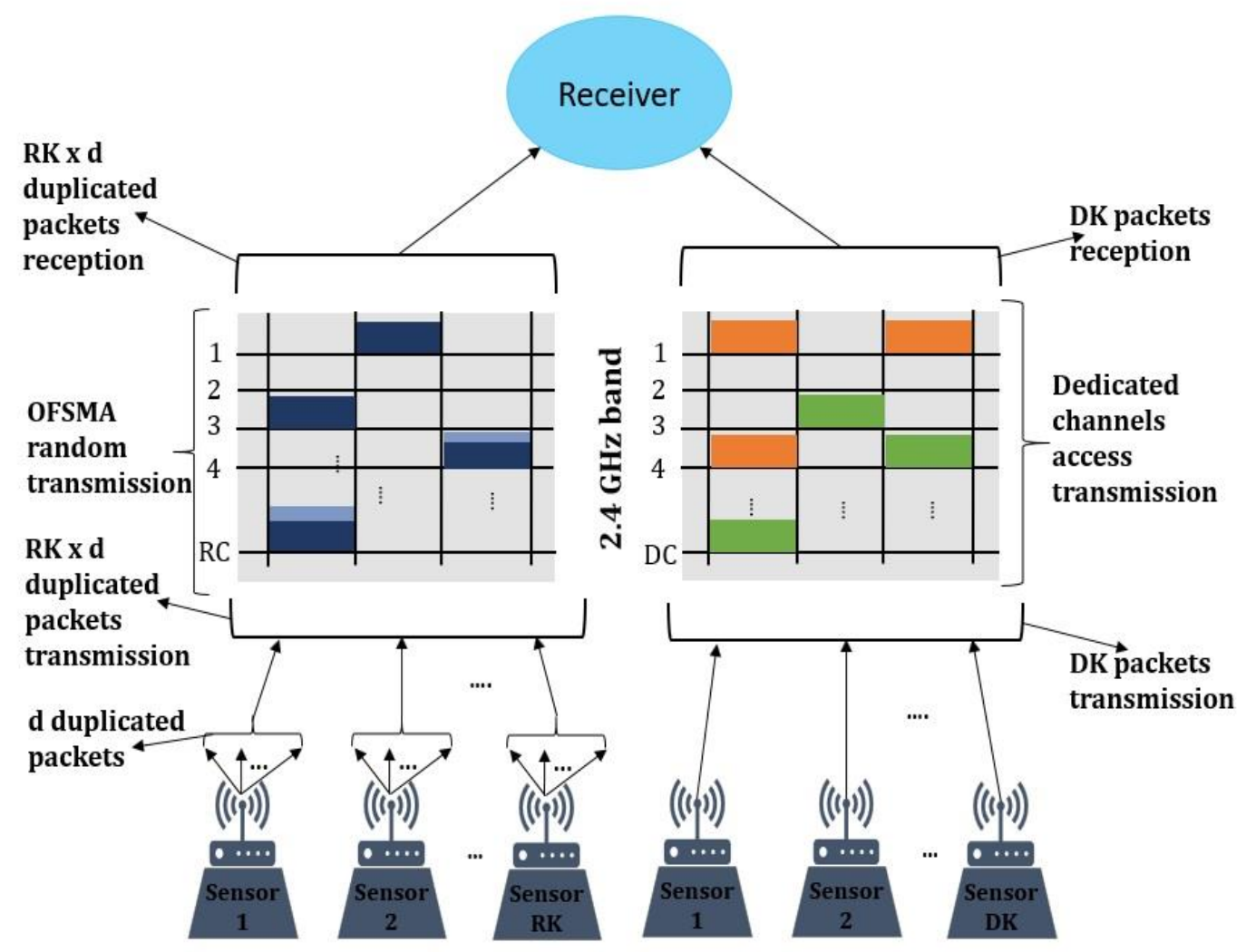

Figure 4. HAS system design

The system assumes a total $K$ number of sensors transmitting over the randomly accessible subcarrier channels defined as RK. The general sensors represented as sensor ${ }_{1}$,

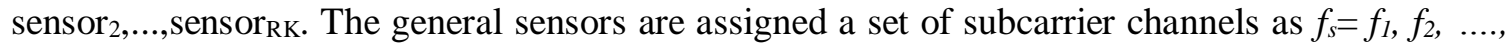
$f_{c}$. Each subcarrier channel $f_{i}$ can be selected randomly as 
International Journal of Computer Networks \& Communications (IJCNC) Vol.12, No.4, July 2020

$$
f_{s} \stackrel{\mathbb{R}}{\leftarrow} f_{i}
$$

Each general sensor transmitting $x=1,2, \ldots, d$ duplicated packets over the randomly selected subcarrier channels $f_{i}$. The $d$ duplicated packet signal $s_{d}(t)$ can be expressed [7] as

$$
S_{d}(t)=\sum_{x=1}^{d} u_{x}(t) e^{j 2 \pi f_{i x} t}
$$

where $u_{x}(t)$ is a complex baseband signal for $x$ number of duplicated packets with an in-phase and quadrature component and $f_{i x}$ is the randomly selected frequency $f_{i}$ for $x$ duplicated packets. The slotted-ALOHA protocol assigns sensors different slot to transmit data packets. For a total of $y=1,2, \ldots, R K$ number of general sensors transmit $d$ duplicated equals to $R K x d$ possible packet can be transmit in a single slot that can be presents as

$$
S_{R K}(t)=\sum_{y=1}^{R K} \sum_{x=1}^{d} u_{x, y}(t) e^{j 2 \pi f_{i x} t}
$$

where $u_{x, y}(t)$ is a complex baseband signal for $x$ number of duplicated packets from $y$ number of sensors having an in-phase and quadrature component. The system also considers a set of audio and video sensors which transmit audio and video packets over the dedicated channels. For a single TTI slot, let's consider a maximum of $D K$ audio or video frames are transmitted over the dedicated subcarrier channel can be expressed as

$$
S_{D K}(t)=\sum_{m=1}^{D K} u(t) e^{j 2 \pi f_{m} t}
$$

Where $\mathrm{u}(\mathrm{t})$ is a complex baseband signal expression for audio or video frame with an in-phase and quadrature component, $f_{m}$ is the dedicated subcarrier frequency channel for the frame and $\mathrm{t}$ determines the duration of the frame. So, the total data transmitted in a single slot over the hybrid access scheme can be represented as

$$
\begin{gathered}
S_{\text {total }}(t)=S_{R K}(t)+S_{D K}(t) \\
S_{\text {total }}(t)=\sum_{y=1}^{R K} \sum_{x=1}^{d} u_{x, y}(t) e^{j 2 \pi f_{i x} t}+\sum_{m=1}^{D K} u(t) e^{j 2 \pi f_{m} t}
\end{gathered}
$$

Equation (5) presents the signal expression for total transmitted packet by the audio, video and general sensors in a single transmission slot. The total signal $S_{\text {total }}(t)$ is transmitted over the AWGN channel, and noise $n(t) \sim C N(0, \sigma 2)$ is added to the signal. For any single TTI slot, the received signal $r_{\text {total }}(t)$ can be defined as

$$
\begin{gathered}
r_{\text {total }}(t)=S_{\text {total }}(t)+n(t) \\
r_{\text {total }}(t)=\sum_{y=1}^{R K} \sum_{x=1}^{d} u_{x, y}(t) e^{j 2 \pi f_{i x} t}+\sum_{m=1}^{D K} u(t) e^{j 2 \pi f_{m} t}+n(t)
\end{gathered}
$$


The equation (6) presents the total received signal with noise added by the receiver. We assumed that, the system has the advanced receiving and decoding capability to receive and decode multiple copies of the data packet at the same time.

\subsection{Signal Propagation}

We consider a short-range signal propagation inside a robot's body. The signal needs to propagation $0.1 \mathrm{~m} \sim 1 \mathrm{~m}$ distance based on sensor deployment over the robot. We consider two types of the signal transmission medium as part of the robotic inner structure in our proposed system, rectangular transmission medium and circular transmission medium presented in Figure 5. The rectangular and circular shaped transmission medium design with IRON metal and outside coated with silver. The inner long tunnel-shaped area of both rectangular and circular shaped transmission medium towards $Z$ - direction is full of air. We assume that the signal transmits from the transmitting point indicated in the Figure and receives at the receiving point defined at the end of the $Z$ - direction. The signal propagates towards $Z$ - direction both for rectangular and circularmedium. The rectangular transmission medium considers three type of width and height configuration as $(190,95) \mathrm{mm},(100,50) \mathrm{mm}$, and $(25,15) \mathrm{mm}$. The circular transmission medium considers three radius configurations as $100 \mathrm{~mm}, 50 \mathrm{~mm}$, and $5 \mathrm{~mm}$. We consider the following parameters listed in Table 1 for evaluating the signal propagation expression of the rectangular and circular shaped transmission medium.

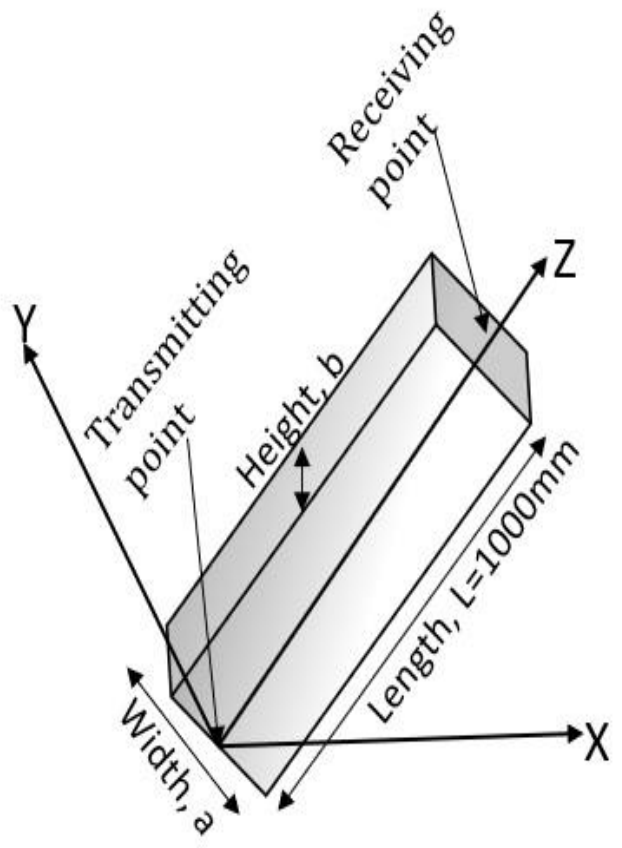

a)

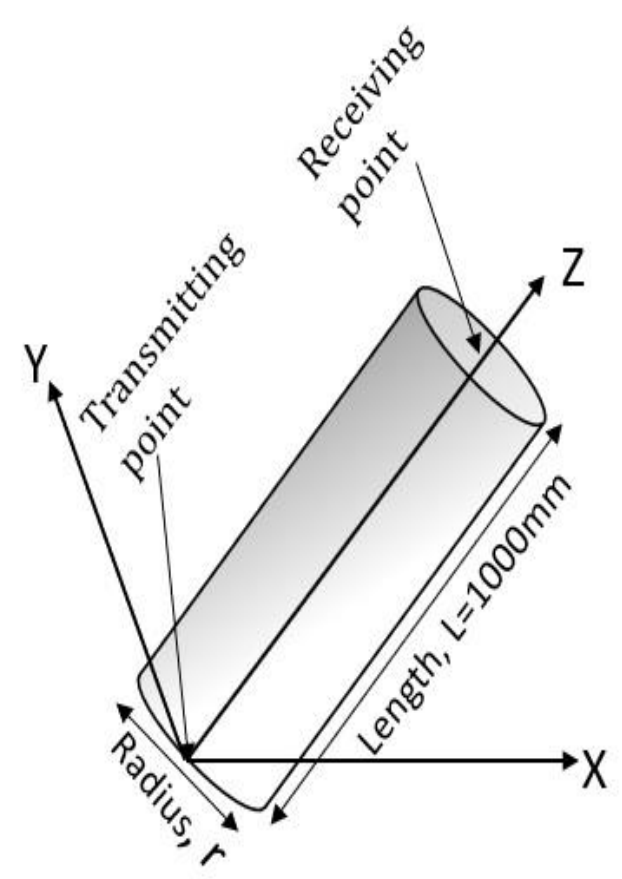

b)

Figure 5. Signal transmission medium, a) Rectangular shaped transmission medium, b) Circular shaped transmission medium. 
International Journal of Computer Networks \& Communications (IJCNC) Vol.12, No.4, July 2020

Table 1. Rectangular and circular transmission medium properties

\begin{tabular}{|c|c|c|c|}
\hline Parameters & \multicolumn{2}{|c|}{$\begin{array}{c}\text { Rectangular transmission } \\
\text { medium }\end{array}$} & Circular transmission medium \\
\hline Transmit power & \multicolumn{2}{|c|}{$20 \mathrm{dBm}$} & $20 \mathrm{dBm}$ \\
\hline Operating frequency & \multicolumn{2}{|c|}{$\begin{array}{c}900 \mathrm{MHz}, 2.4 \mathrm{GHz}, 24 \mathrm{GHz} \text {, and } \\
55 \mathrm{GHz}\end{array}$} & $\begin{array}{l}900 \mathrm{MHz}, 2.4 \mathrm{GHz}, 24 \mathrm{GHz} \text {, and } \\
55 \mathrm{GHz}\end{array}$ \\
\hline \multirow{4}{*}{ Structure } & width & Height & Radius \\
\hline & $190 \mathrm{~mm}$ & $95 \mathrm{~mm}$ & $100 \mathrm{~mm}$ \\
\hline & $100 \mathrm{~mm}$ & $50 \mathrm{~mm}$ & $50 \mathrm{~mm}$ \\
\hline & $25 \mathrm{~mm}$ & $15 \mathrm{~mm}$ & $5 \mathrm{~mm}$ \\
\hline Length & \multicolumn{2}{|c|}{$1000 \mathrm{~mm}$} & $1000 \mathrm{~mm}$ \\
\hline Delta S & \multicolumn{2}{|c|}{0.02} & 0.02 \\
\hline Relative permittivity, air & \multicolumn{2}{|c|}{1.0006} & 1.0006 \\
\hline Relative permittivity, iron & \multicolumn{2}{|c|}{1} & 1 \\
\hline Relative permittivity, silver & \multicolumn{2}{|c|}{1} & 1 \\
\hline Relative permeability, air & \multicolumn{2}{|c|}{1.0000004} & 1.0000004 \\
\hline Relative permeability, iron & \multicolumn{2}{|c|}{4000} & 4000 \\
\hline Relative permeability, silver & \multicolumn{2}{|c|}{0.99998} & 0.99998 \\
\hline
\end{tabular}

\section{Simulation Results}

This section covers the details of our proposed $H A S$ system simulation and performance evaluation. We simulate our HAS system in a system-level simulation generating in the MATLAB simulator to evaluate the performance of our hybrid access scheme. The signal propagation expression is captured using ANSYS HFSS software. The 90 sensors transmitted 3packet, 5-, and 7- packet duplication over a different number of subcarrier channels where the subcarrier selection is random and determine the minimum number of subcarriers, reliability and collision probability of the system. We consider random subcarrier selection in order to minimize the scheduling latency of the system. The packet generation and transmission follow the poison arrival process. The sensors transmit packets over a slotted ALOHA system and each slot duration is $0.1 \mathrm{~ms}$. The HAS system considers BPSK modulation scheme to ensure low bit error of the transmitted packets. The simulation runs for 1000s and presented in the figure. The details simulation parameters are presented in Table 2.

\subsection{Reliability and Collision Probability}

The 90 sensors transmit 3-, 5-, and 7-packet duplication over $2.4 \mathrm{GHz}$ frequency band and determine the minimum number of subcarriers to satisfy the URLLC defined reliability of 99.999\% for different arrival conditions presented in Figure 6. The 3-packet duplication secured reliability of $99.999 \%$ having a higher number of subcarriers included in the system compared to all other packet duplications. At an arrival rate of $5000 \mathrm{pk} / \mathrm{sec}$, the 3-packet duplications demanded 440 subcarriers to satisfy the reliability of $99.999 \%$ for packet transmission. This happens due to the smaller number of packet duplications and a higher number of intra-packet 
International Journal of Computer Networks \& Communications (IJCNC) Vol.12, No.4, July 2020

collisions. The 5-packet duplication secured $99.999 \%$ reliability with a lower number of subcarriers demands compared to 3-packet duplications but higher than 7-packet duplications. At $5000 \mathrm{pk} / \mathrm{sec}$ arrival condition, the 5-packet duplications required 140 subcarriers to satisfy the reliability of $99.999 \%$ for packet transmission. The 7-packet duplication achieved $99.999 \%$ reliability having the lower number of subcarriers demands compared to all other packet duplications. At $5000 \mathrm{pk} / \mathrm{sec}$, the 7-packet duplications demanded 110 subcarriers to satisfy the URLLC defined reliability of $99.999 \%$ for packet transmission.

Table 2. Simulation properties

\begin{tabular}{|c|c|}
\hline Parameters & Values \\
\hline Total general sensor & 90 \\
\hline Total random Subcarrier channel & $60 \sim 450$ \\
\hline Total audio Sensor & 5 \\
\hline Total video sensor & 5 \\
\hline Dedicated channel & 10 \\
\hline Carrier frequency & $2.4 \mathrm{GHz}$ \\
\hline Operating frequency for signal & $900 \mathrm{MHz}, 2.4 \mathrm{GHz}, 24 \mathrm{GHz}$, and $55 \mathrm{GHz}$ \\
\hline expression & $10 \mathrm{KHz}$ \\
\hline Subcarrier bandwidth & $100 \mathrm{bits}$ \\
\hline Packet size & $\mathrm{BPSK}$ \\
\hline Modulation & $20 \mathrm{dBm}$ \\
\hline Transmit Power & $1 \mathrm{Mbps}$ \\
\hline Link speed & $3,5 \mathrm{and} 7$ \\
\hline Packet duplication & $1 \sim 10000 \mathrm{pk} / \mathrm{sec}$ \\
\hline Arrival rate, $\lambda$ & $0.1 \mathrm{~ms}$ \\
\hline Slot duration & $1000 \mathrm{~s}$ \\
\hline Simulation time & \\
\hline & \\
\hline & \\
\hline & \\
\hline & \\
\hline & \\
\hline & \\
\hline & \\
\hline & \\
\hline & \\
\hline &
\end{tabular}




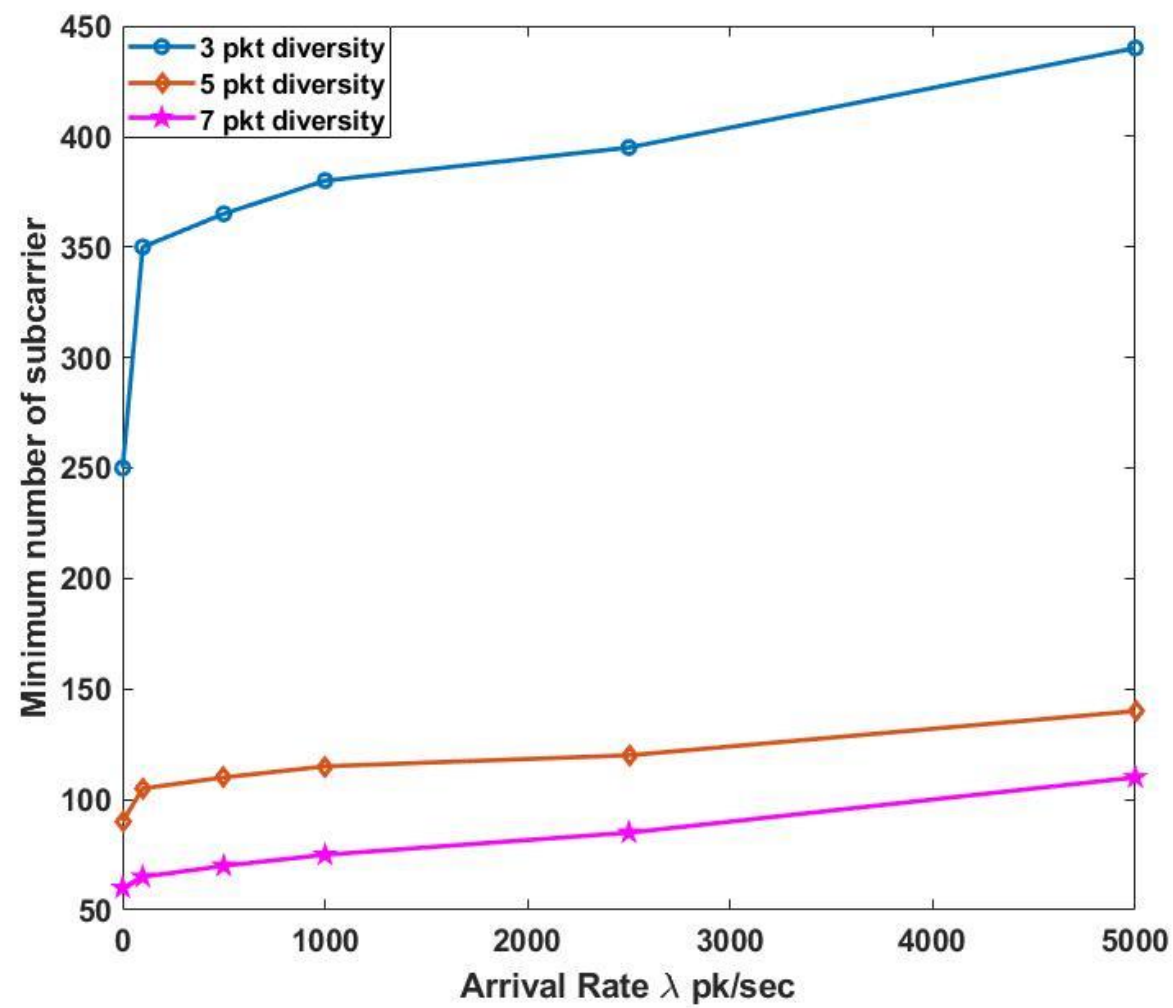

Figure 6. The minimum number of subcarriers determined for satisfying URLLC reliability of $99.999 \%$ for different packet duplication over $2.4 \mathrm{GHz}$ frequency band.

The reliability and collision probability is measured for all sensors. The 3-, 5-, and 7-packet duplications transmitted packets over randomly selected 90 subcarrier channels. The audio and video sensors transmitted a single packet over the 10 dedicated channels. At the receiver side, we evaluated the reliability and collision probability for audio, video and general sensors. The reliability for 3-, 5-, 7-packet duplication and dedicated channel assigned audio and video sensors is presented in Figure 7.

As shown in Figure 7, the 3-packet duplication has lower reliability compared to all others in the simulation results due to small number of duplicated packets and subcarrier channels. At an arrival rate of $10000 \mathrm{pk} / \mathrm{sec}$, the 3-packet duplication reliability is $99.9578 \%$. The 3-packet duplication could not achieve $99.999 \%$ reliability for any arrival conditions due to small number of packet duplications. The 5-packet duplication has higher reliability compared to 3-packet duplication but lower than 7-packet and dedicated channels accessible audio and video sensors. At arrival of $1 \mathrm{pk} / \mathrm{sec}$, the 5-packet has secured 99.9995\% reliability and at the highest arrival rate $10000 \mathrm{pk} / \mathrm{sec}$ the reliability is $99.9867 \%$. The 10 audio and video sensors reliability response are closed to 7-packet duplication reliability up to $2500 \mathrm{pk} / \mathrm{sec}$ arrival rates after that the dedicated channels improved its reliability response. The dedicated channels reliability response and 7packet reliability response are zoomed from $1000 \mathrm{pk} / \mathrm{sec}$ to $10000 \mathrm{pk} / \mathrm{sec}$ arrival rates. The 7packet secure $99.9994 \%$ reliability at an arrival rate of $2500 \mathrm{pk} / \mathrm{sec}$ but after that arrival rate its reliability is decreased and lowers than $99.999 \%$. At an arrival rate of $10000 \mathrm{pk} / \mathrm{sec}$, the 7-packet reliability response is $99.989 \%$. The 10 audio and video sensors dedicated channel evaluated 99.999\% reliability up to arrival rate $7500 \mathrm{pk} / \mathrm{sec}$ and at an arrival rate of $10000 \mathrm{pk} / \mathrm{sec}$, the estimated reliability is $99.9989 \%$. 


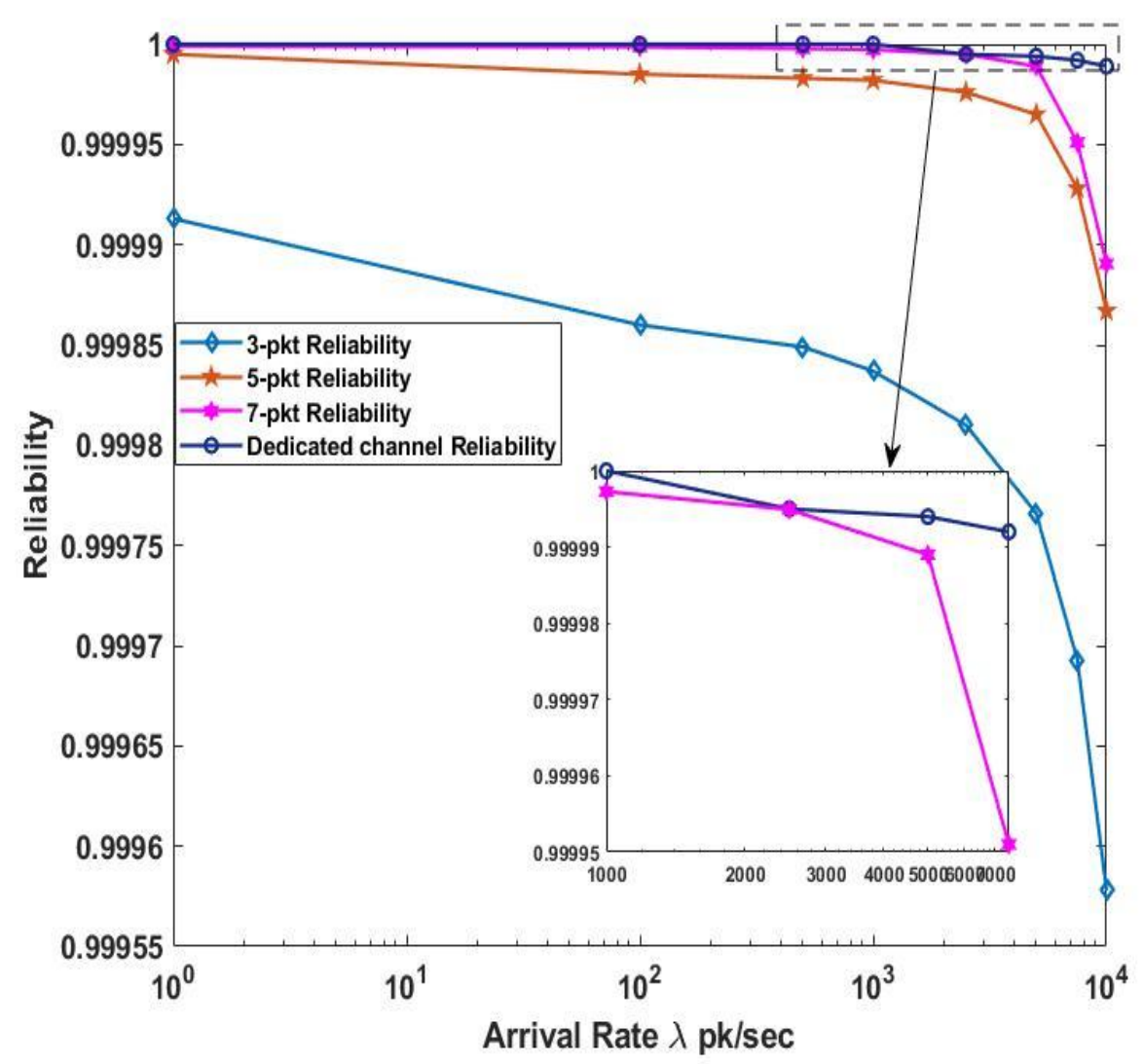

Figure 7. Reliability response for 3-packet, 5-packet, 7-packet and 10 audio and video sensors dedicated channel.

The 90 general sensors transmit 3-, 5-, 7-packet duplication over the 90 subcarrier channels and 10 audio and video sensors forward a single packet over 10 dedicated channels. At the receiver site, we evaluate the collision probability that presented in Figure 8. According to Figure 8, the 3packet duplication has a higher collision probability than 5-, and 7-packet duplications due to lower number of packet duplications and a small number of subcarrier channels. At an arrival rate of $10000 \mathrm{pk} / \mathrm{sec}$, the 3-packet duplications collision probability is $0.0422 \%$. The 5-packet duplications collision probability is lower than 3 -packet duplications but higher than 7-packet duplications. At the highest $10000 \mathrm{pk} / \mathrm{sec}$ arrival rate, the 5-packet collision probability is $0.0133 \%$. The 7-packet duplications collision probability is higher than 3-, and 5-packet duplications and at arrival rate of $10000 \mathrm{pk} / \mathrm{sec}$ it achieved $0.011 \%$. Among the audio and video sensors transmitted packets, there is no collision but very few packets lost due to low power at the receiver. We set the minimum threshold power to receive at receiver is $39.798 \mathrm{dBm}$ for single $2.4 \mathrm{GHz}$ frequency band. The packets having lower power than that is dropped, and the collision probability is presented along with 7-packet duplication inside the zoom section. The audio and video sensors secured lower collision probability than all other comparisons and at an arrival rate of $10000 \mathrm{pk} / \mathrm{sec}$, it secured $0.0011 \%$. The HAS system assumed that there is no collision among the duplicated packets emitted from same source in the network. Moreover, in a sequence of bitstreams, if there is a combination of success packets and collide packets bits, the receiver assumed to decode and select one the success packets bit sequence from the total bit sequence. 


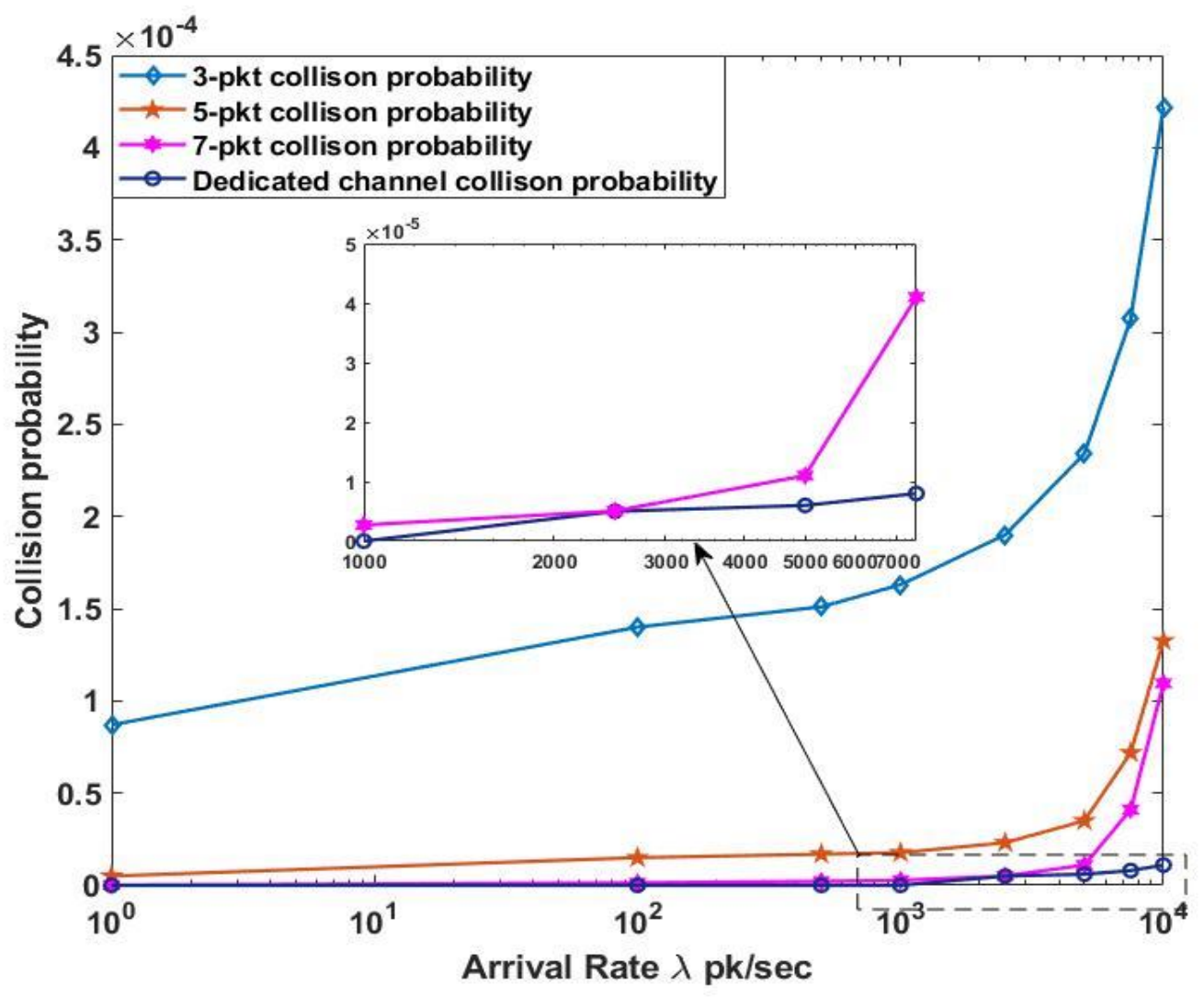

Figure 8. Collision probability for 3-packet, 5-packet, 7-packet and 10 audio and video sensors dedicated channel.

\subsection{Signal Propagation}

The signal expressions are captured for rectangular and circular transmission medium at maximum 1 meter $(1000 \mathrm{~mm})$ distance as the different internal structure of a future robot body. The signal propagation is measured and captured for $900 \mathrm{MHz}, 2.4 \mathrm{GHz}, 24 \mathrm{GHz}$, and $55 \mathrm{GHz}$ frequency bands. The measurement properties are depicted in Table 1. The rectangular transmission medium having width of $190 \mathrm{~mm}$ and height of $95 \mathrm{~mm}$ signal expression captured for defined frequency bands that presented in Figure 9. The signal is transmitted through the air medium to $Z$ direction at about $1000 \mathrm{~mm}$ long. For the rectangular transmission medium having a configuration of width $190 \mathrm{~mm}$, and height $95 \mathrm{~mm}$, the strong signal is transmitted over the 900 $\mathrm{MHz}$ and $2.4 \mathrm{GHz}$ frequency bands. The received signal is comparatively weak for $24 \mathrm{GHz}$ frequency band and for $55 \mathrm{GHz}$ the signal could not propagate at the end towards the receiver to $Z$ - direction.

The signal propagation expression for rectangular transmission medium with a width of $100 \mathrm{~mm}$ and a height of $50 \mathrm{~mm}$ is presented in Figure 10. The signal could not propagate through 900 $\mathrm{MHz}, 24 \mathrm{GHz}$, and $55 \mathrm{GHz}$ frequency bands at the end of the $Z$ - direction to the receiver due to half of the size of the rectangular medium. But only $2.4 \mathrm{GHz}$ frequency bands successfully propagated the signal at the receiving $Z$ - direction. Moreover, the signal slightly propagated over $900 \mathrm{MHz}$ is comparatively strong compared to $24 \mathrm{GHz}$ frequency band and the signal propagated over $24 \mathrm{GHz}$ frequency band is also comparatively strong compared to $55 \mathrm{GHz}$ frequency band. 


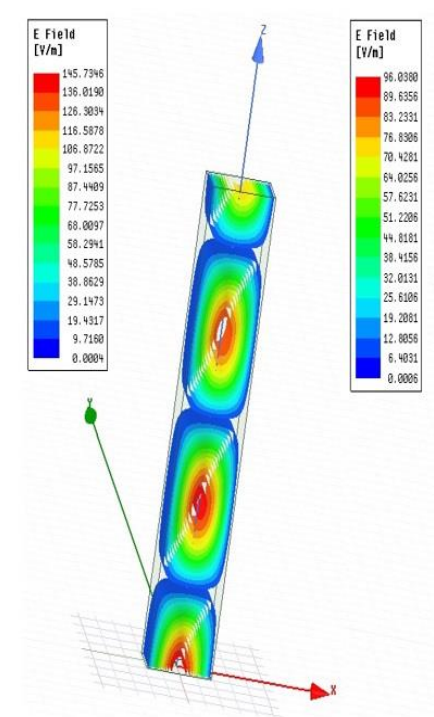

a) $900 \mathrm{MHz}$

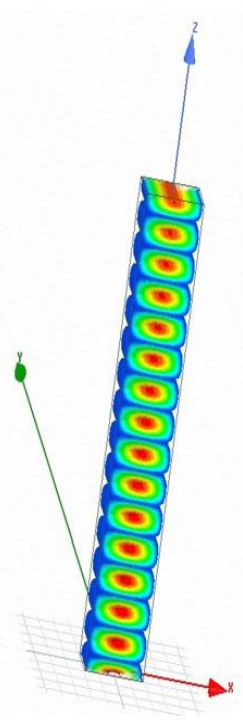

b) $2.4 \mathrm{GHz}$

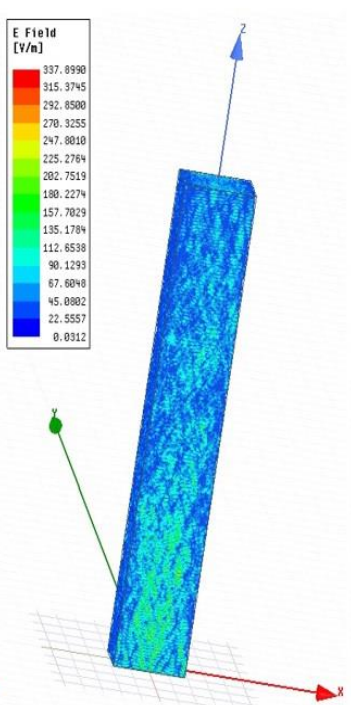

c) $24 \mathrm{GHz}$

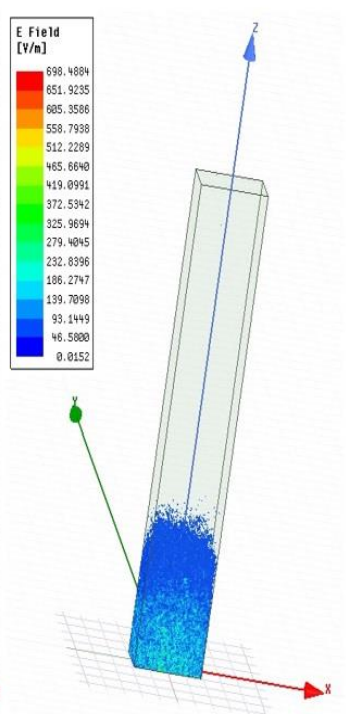

d) $55 \mathrm{GHz}$

Figure 9. Signal propagation over $1000 \mathrm{~mm}$ long, $195 \mathrm{~mm}$ width and $95 \mathrm{~mm}$ height rectangular transmission medium for, a) $900 \mathrm{MHz}$ frequency band b) $2.4 \mathrm{GHz}$ frequency band, c) $24 \mathrm{GHZ}$ frequency band, and d) $55 \mathrm{GHz}$ frequency band.

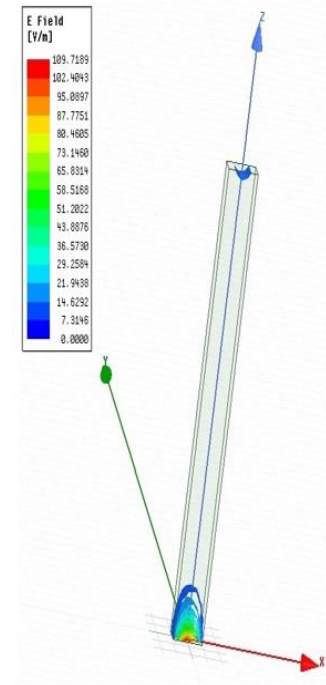

a) $900 \mathrm{MHz}$

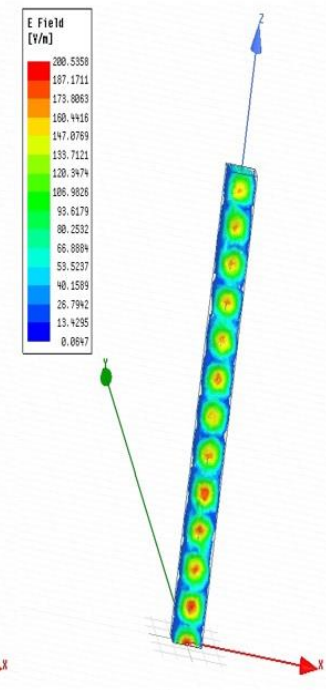

b) $2.4 \mathrm{GHz}$

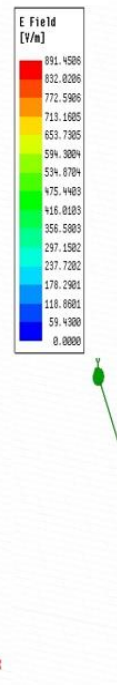

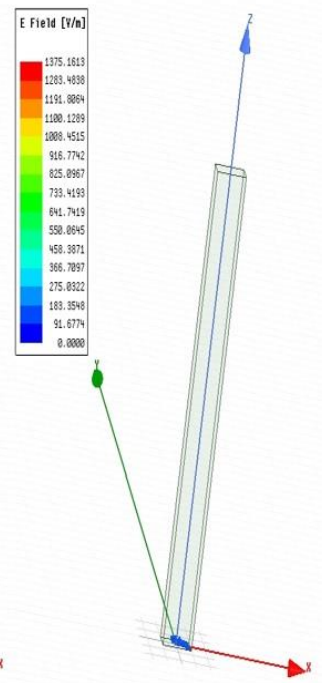

d) $55 \mathrm{GHz}$

Figure 10. Signal propagation over $1000 \mathrm{~mm}$ long, $100 \mathrm{~mm}$ width and $50 \mathrm{~mm}$ height rectangular transmission medium for, a) $900 \mathrm{MHz}$ frequency band b) $2.4 \mathrm{GHz}$ frequency band, c) $24 \mathrm{GHZ}$ frequency band, and d) $55 \mathrm{GHz}$ frequency band.

For the width of $25 \mathrm{~mm}$ and a height of $15 \mathrm{~mm}$ of the rectangular transmission medium signal expression is illustrated in Figure 11. The $900 \mathrm{MHz}$ and $2.4 \mathrm{GHz}$ frequency band shown a dim signal expression, but $24 \mathrm{GHz}$ and $55 \mathrm{GHz}$ present a strong signal propagation compared to previous rectangular configuration. By analyzing the signal propagation expression presented in Figure 9-11, the higher size of width and height is better for lower frequency bands and the lower width and height size shown a comparatively better signal propagation expression for higher frequency bands. 


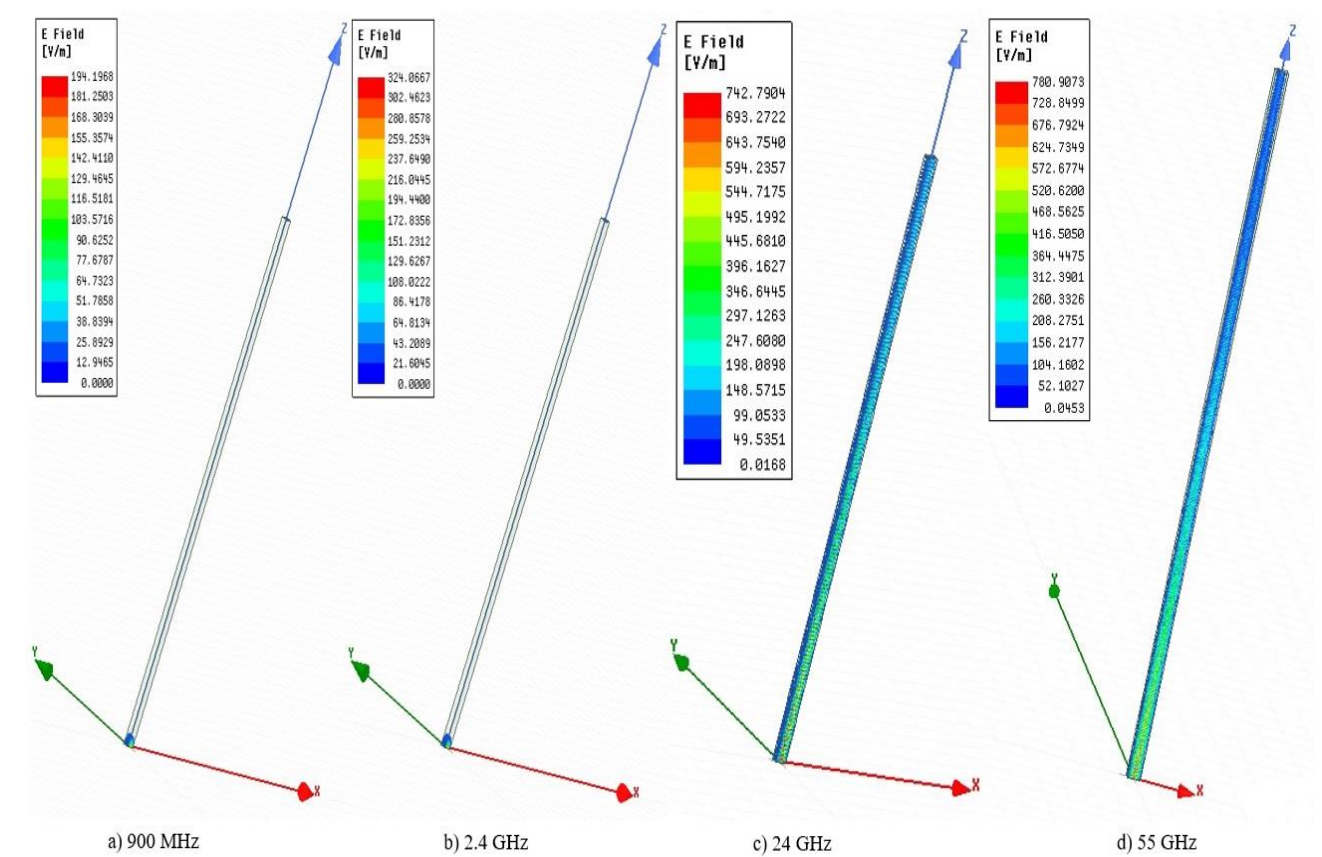

Figure 11. Signal propagation over $1000 \mathrm{~mm}$ long, $25 \mathrm{~mm}$ width and $15 \mathrm{~mm}$ height rectangular transmission medium for, a) $900 \mathrm{MHz}$ frequency band b) $2.4 \mathrm{GHz}$ frequency band, c) $24 \mathrm{GHZ}$ frequency band, and d) $55 \mathrm{GHz}$ frequency band.

The signal propagation for a circular transmission medium is illustrated in the figure 12-14. The circular transmission medium having a radius of $100 \mathrm{~mm}$ is presented in the figure 12 . As presented in figure, the signal is successfully propagated over $900 \mathrm{MHz}$ and $2.4 \mathrm{GHz}$ frequency bands to the receiver towards $Z$ - direction and signal strength is strong enough. The signal could not propagate to the receiver over $24 \mathrm{GHz}$ and $55 \mathrm{GHz}$ frequency bands and signal strength is extremely low compared to $900 \mathrm{MHz}$ and $2.4 \mathrm{GHz}$ frequency bands.

The signal propagation for circular transmission medium having radius of $50 \mathrm{~mm}$ is presented in Figure 13. The signal only propagated over $2.4 \mathrm{GHz}$ frequency band and none of the other three frequency band could not propagate the signal to the receiver towards $Z$ - direction. Moreover, the $55 \mathrm{GHz}$ frequency band's propagate signal is the weakest signal compared to all others. So, for the robotic internal transmission, the proposed circular structure performance is worse compare to other similar structures.

The signal propagation expression illustrated in Figure 14 for $5 \mathrm{~mm}$ radius shown the signal is transmitted to the receiver towards $Z$ direction for $24 \mathrm{GHz}$ and $55 \mathrm{GHz}$ but a very poor signal expression for $900 \mathrm{MHz}$ and $2.4 \mathrm{GHz}$ frequency bands. This circular structure shows better signal strength for $24 \mathrm{GHz}$ and $55 \mathrm{GHz}$ frequency bands compared to the previous circular transmission medium structure. That implies this structure is recommended as better for higher frequency bands. The circular transmission medium is analyzed for three different radius configurations over $900 \mathrm{MHz}, 2.4 \mathrm{GHz}, 24 \mathrm{GHz}$, and $55 \mathrm{GHz}$ frequency bands. The higher radius configuration of the circular transmission medium is more capable to transmit strong signals and able to propagate signals to the receiver towards $Z$ - direction for comparatively lower frequency bands. On the other hands, the lower circular transmission medium structure viable for higher frequency bands with strong signals. 
International Journal of Computer Networks \& Communications (IJCNC) Vol.12, No.4, July 2020

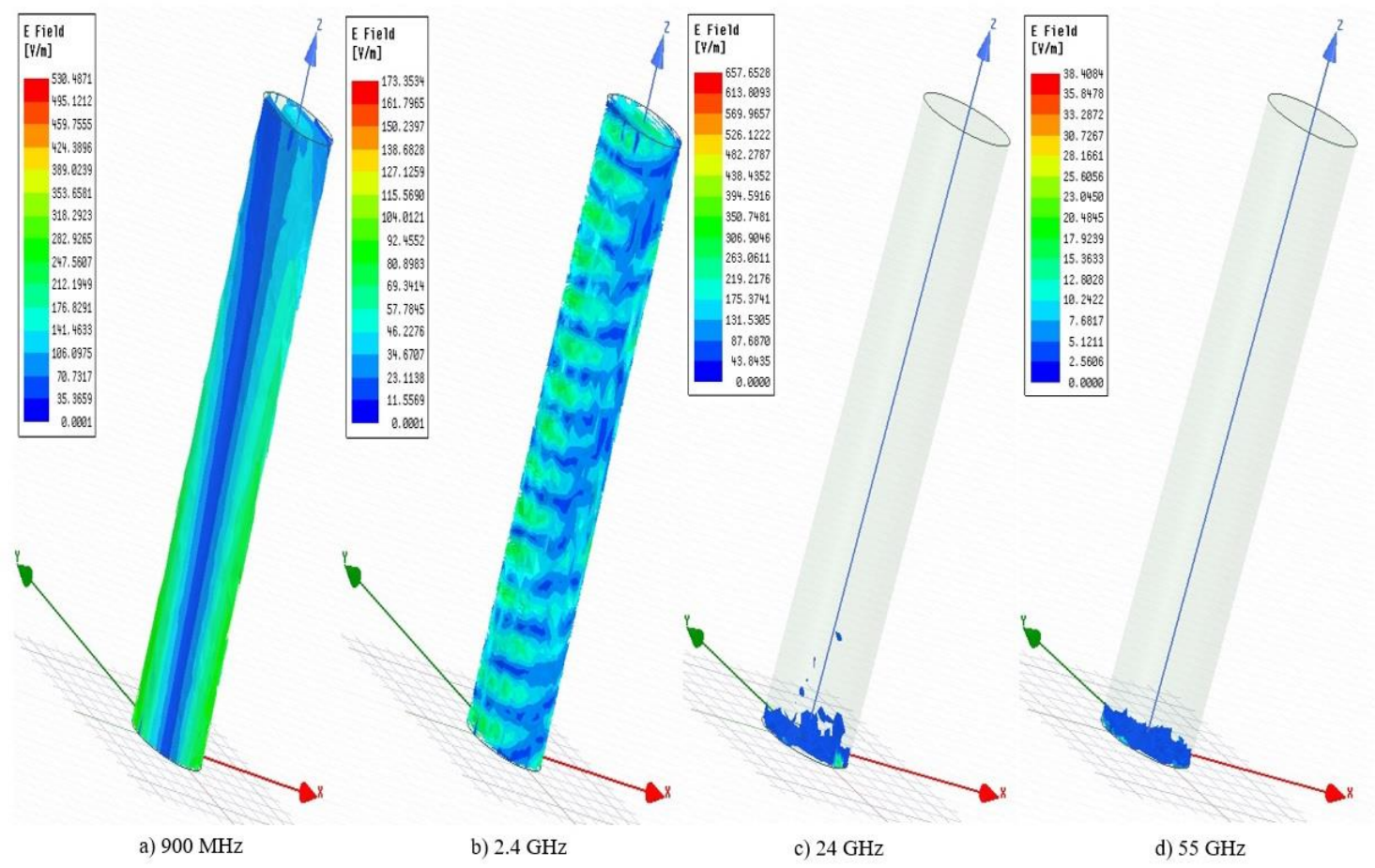

Figure 12. Signal propagation over $1000 \mathrm{~mm}$ long, and $100 \mathrm{~mm}$ radius circular transmission medium for, a) $900 \mathrm{MHz}$ frequency band b) $2.4 \mathrm{GHz}$ frequency band, c) $24 \mathrm{GHZ}$ frequency band, and d) $55 \mathrm{GHz}$ frequency band.

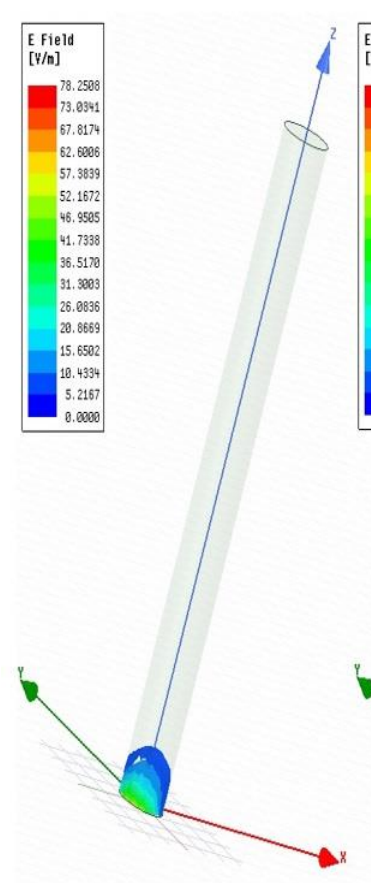

a) $900 \mathrm{MHz}$

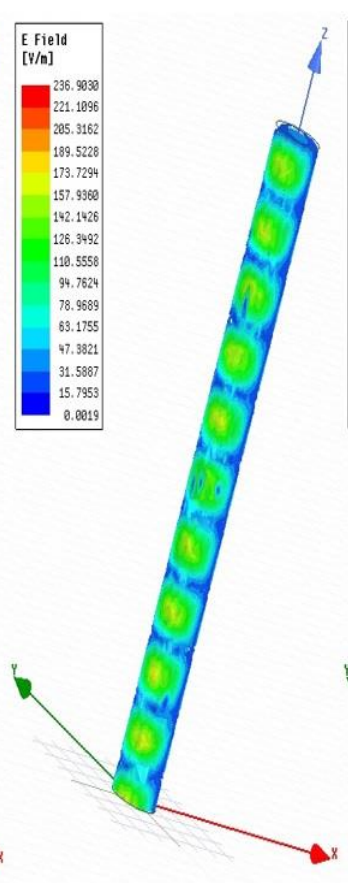

b) $2.4 \mathrm{GHz}$

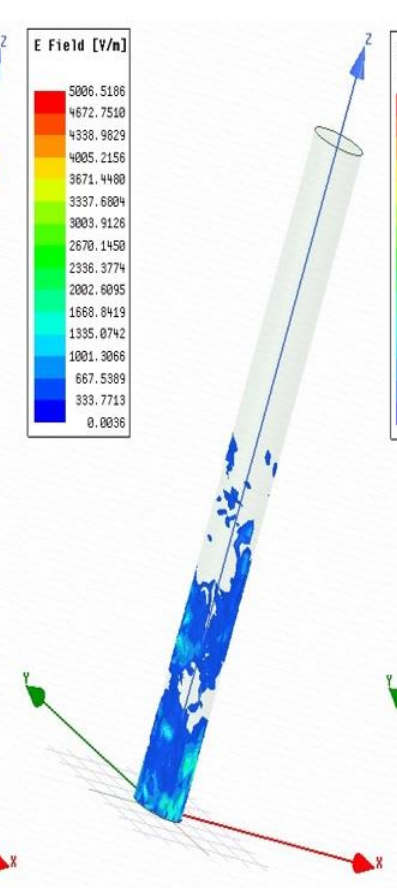

c) $24 \mathrm{GHz}$

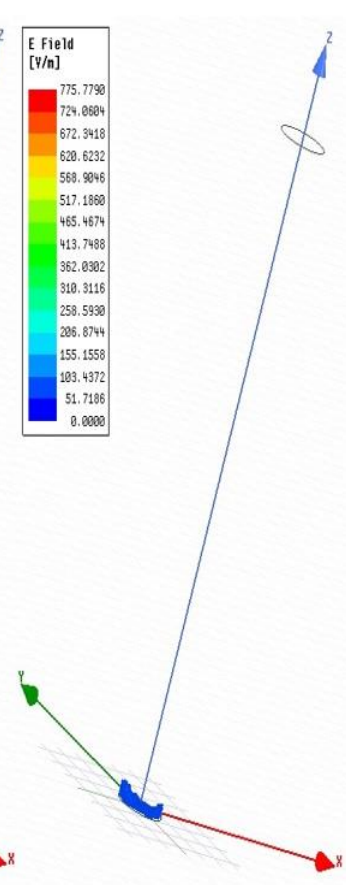

d) $55 \mathrm{GHz}$

Figure 13. Signal propagation over $1000 \mathrm{~mm}$ long, and $50 \mathrm{~mm}$ radius circular transmission medium for, a) $900 \mathrm{MHz}$ frequency band b) $2.4 \mathrm{GHz}$ frequency band, c) $24 \mathrm{GHZ}$ frequency band, and d) $55 \mathrm{GHz}$ frequency band. 


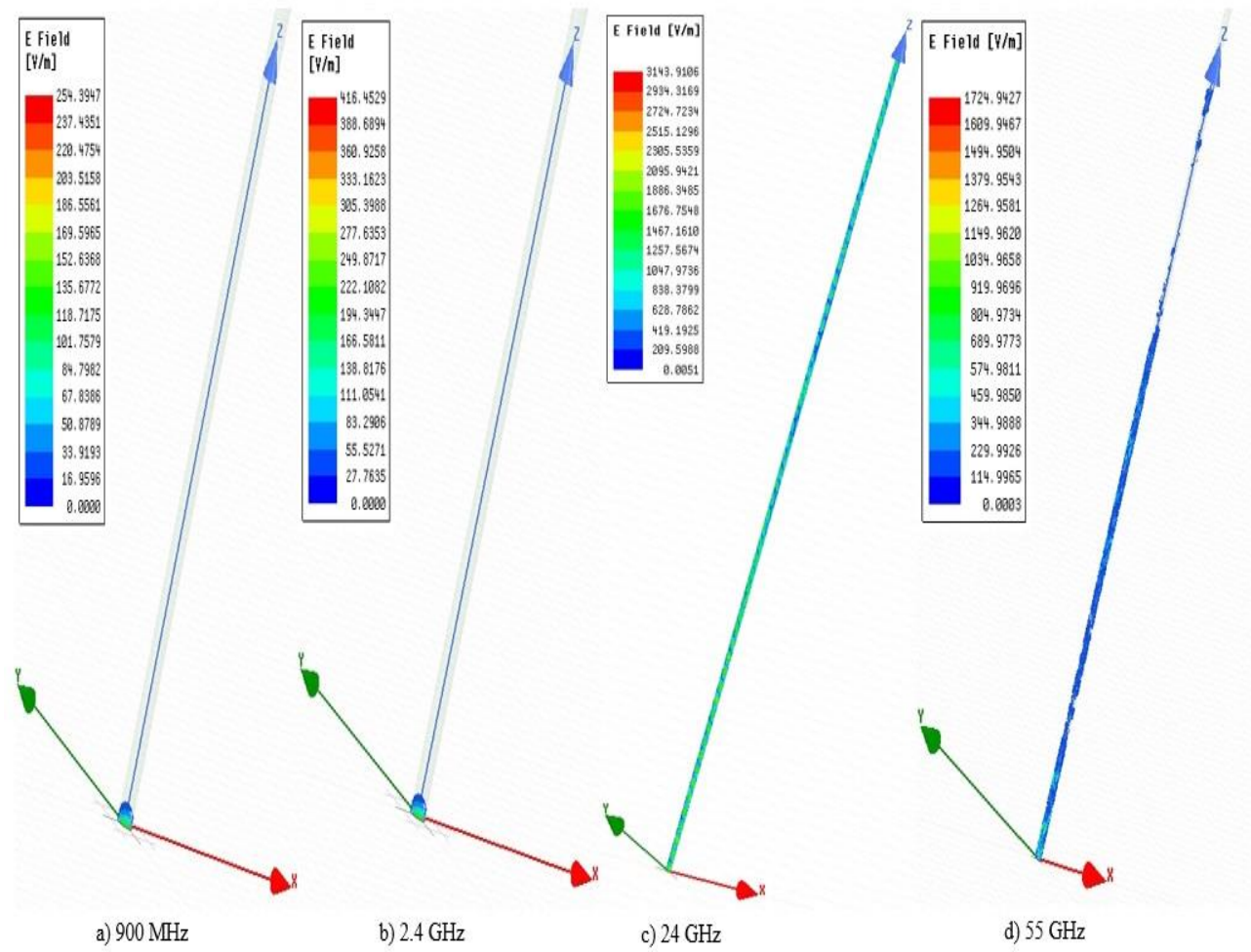

Figure 14. Signal propagation over $1000 \mathrm{~mm}$ long, and $5 \mathrm{~mm}$ radius circular transmission medium for, a) $900 \mathrm{MHz}$ frequency band b) $2.4 \mathrm{GHz}$ frequency band, c) $24 \mathrm{GHZ}$ frequency band, and d) $55 \mathrm{GHz}$ frequency band.

The signal propagation expression results for different frequency bands and different structural configuration is summarized in Table 3 with an overview defining either the signal is strong, average or bad over $900 \mathrm{MHz}, 2.4 \mathrm{GHz}, 24 \mathrm{GHz}$, and $55 \mathrm{GHz}$ frequency bands both for the rectangular and circular transmission medium. Moreover, the result presented in Table 3 also defines either the signal is propagated to the receiving point towards $Z$ for different frequency bands and different structural configurations or not.

\section{Conclusion}

In this paper, a hybrid access scheme is proposed for future robot's internal communication by sensors instead of wire. The audio, video and general sensors able to transmit signals simultaneously over the hybrid access scheme. The audio and video sensors transmit signals over the dedicated subcarrier channels and the general sensors transmit signals over the randomly selected subcarrier channels. The OFSMA system's reliability is evaluated for $99.999 \%$ as URLLC reliability requirement and determine the minimum number of subcarriers demanded to achieve this reliability level for different packet duplications. The 3-packet duplication needed 440 subcarriers, 5-packet duplication demanded 140 subcarriers, and finally the 7-packet duplications required 110 subcarriers to satisfy the URLLC recommended reliability of $99.999 \%$ at $5000 \mathrm{pk} / \mathrm{sec}$ arrival conditions. The HAS system's reliability and collision probability is evaluated for fixed channel conditions and determine either different arrival conditions the reliability satisfies the URLLC specified reliability requirement or not. For fixed channel assignment, the URLLC reliability requirement of 99.999\%, 3-packet duplication not satisfied for 
International Journal of Computer Networks \& Communications (IJCNC) Vol.12, No.4, July 2020

Table 3. Signal propagation results for different structural configuration over different frequency bands

\begin{tabular}{|c|c|c|c|c|c|c|c|c|c|c|}
\hline & \multicolumn{2}{|c|}{ Parameters } & \multicolumn{2}{|c|}{$900 \mathrm{MHz}$} & \multicolumn{2}{|c|}{$2.4 \mathrm{GHz}$} & \multicolumn{2}{|c|}{$24 \mathrm{GHz}$} & \multicolumn{2}{|c|}{$55 \mathrm{GHz}$} \\
\hline \multirow{4}{*}{ 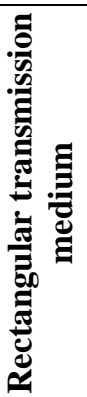 } & Width & Height & Strength & $\begin{array}{c}\text { Propaga } \\
\text { te? }\end{array}$ & Strength & $\begin{array}{c}\text { Propagate } \\
?\end{array}$ & Strength & $\begin{array}{c}\text { Propaga } \\
\text { te? }\end{array}$ & Strength & $\begin{array}{c}\text { Propagate } \\
?\end{array}$ \\
\hline & $190 \mathrm{~mm}$ & $95 \mathrm{~mm}$ & Strong & Yes & Strong & Yes & Average & Yes & $\mathrm{Bad}$ & No \\
\hline & $100 \mathrm{~mm}$ & $50 \mathrm{~mm}$ & $\mathrm{Bad}$ & No & Strong & Yes & $\mathrm{Bad}$ & No & $\mathrm{Bad}$ & No \\
\hline & $25 \mathrm{~mm}$ & $15 \mathrm{~mm}$ & $\mathrm{Bad}$ & No & Bad & No & Strong & Yes & Average & yes \\
\hline \multirow{3}{*}{ 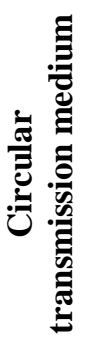 } & \multirow{3}{*}{ 节 } & $100 \mathrm{~mm}$ & Strong & Yes & Strong & Yes & Bad & No & $\mathrm{Bad}$ & No \\
\hline & & $50 \mathrm{~mm}$ & $\mathrm{Bad}$ & No & Strong & Yes & Bad & No & $\mathrm{Bad}$ & No \\
\hline & & $5 \mathrm{~mm}$ & $\mathrm{Bad}$ & No & Bad & No & Strong & Yes & Average & Yes \\
\hline
\end{tabular}

any arrival condition, 5-packet duplication satisfied for $1 \mathrm{pk} / \mathrm{sec}$, 7-packet duplication satisfied for up to $2500 \mathrm{pk} / \mathrm{sec}$, and finally dedicated channel assignment satisfied for $7500 \mathrm{pk} / \mathrm{sec}$ arrival condition. Moreover, the signal propagation initiated by different sensors is also simulated over ANSYS HFSS software for $900 \mathrm{MHz}, 2.4 \mathrm{GHz}, 24 \mathrm{GHz}$ and $55 \mathrm{GHz}$ frequency bands. The transmission medium having size (width, height and radius) of higher number is shown better performance for comparatively lower frequency bands and vice-versa. For signal measurement, we cannot determine a fixed value of received power in $\mathrm{dBm}$ in the receiving point. In future, we try to resolve the problem. The system is simulated over two different simulation platform but in real field the result might have more interference and the result might be affected by different factors.

We investigate our system for a short-range robotic communication system. The HAS scheme might also applicable for any short-range, mission-critical, higher reliable system. The HAS system might also consider automation of vehicles and other types of robotic or automated communication domains.

\section{CONFLICTS OF INTEREST}

The authors declare no conflict of interest.

\section{ACKNOWLEDGMENT}

This research work is supported by JICA INNOVATIVE ASIA program. We grateful to JICA. 
International Journal of Computer Networks \& Communications (IJCNC) Vol.12, No.4, July 2020

\section{REFERENCES}

[1] Shimojo, M., Araki, T., Teshigawara, S., Ming, A., \& Ishikawa, M. (2007, October). A net-structure tactile sensor covering free-form surface and ensuring high-speed response. In 2007 IEEE/RSJ International Conference on Intelligent Robots and Systems (pp. 670-675). IEEE.

[2] Ganjalizadeh, M., Di Marco, P., Kronander, J., Sachs, J., \&Petrova, M. (2019, December). Impact of correlated failures in 5G dual connectivity architectures for URLLC applications. In 2019 IEEE Globecom Workshops (GC Wkshps) (pp. 1-6). IEEE. [3]3GPP, “5G; Study on Scenarios and Requirements for Next Generation Access Technologies,” Tech. Rep., 38.913,14.3.0, 2017.

[4] D’Errico, L., Franchi, F., Graziosi, F., Marotta, A., Rinaldi, C., Boschi, M., \&Colarieti, A. (2019, April). Structural Health Monitoring and Earthquake Early Warning on 5G uRLLC Network. In 2019 IEEE 5th World Forum on Internet of Things (WF-IoT) (pp. 783-786). IEEE.

[5] Notay, J. K., \& Safdar, G. A. (2011, September). A wireless sensor network based structural health monitoring system for an airplane. In The 17th International Conference on Automation and Computing (pp. 240-245). IEEE.

[6] Almarashdeh, I., Alsmadi, M., Hanafy, T., Albahussain, A., Altuwaijri, N., Almaimoni, H., ... \& Al Fraihet, A. (2018). Real-time elderly healthcare monitoring expert system using wireless sensor network. International Journal of Applied Engineering Research ISSN, 0973-4562.

[7] Ratiu, O., Panagiotopoulos, N., Vos, S., \& Puschita, E. (2018, December). Wireless Transmission of Sensor Data over UWB in Spacecraft Payload Networks. In 2018 6th IEEE International Conference on Wireless for Space and Extreme Environments (WiSEE) (pp. 131-136). IEEE.

[8] M. A. Hossain, M. Saitou, Z. Pan, J. Liu, and S. Shimamoto. (2020) "Orthogonal Frequency Subcarrier-based Multiple Random Access in Ultra Reliability and Low Latency Communication," in IEEE Consumer Communications Networking Conference, ser. 2nd IEEE Workshop on CyberPhysical Networking (CPN'20), In press

[9] Islam, A., Musavian, L., \& Thomos, N. (2019, December). Performance Analysis of Vehicular Optical Camera Communications: Roadmap to uRLLC. In 2019 IEEE Global Communications Conference (GLOBECOM) (pp. 1-6). IEEE.

[10] Yoshizawa, T., Baskaran, S. B. M., \& Kunz, A. (2019, October). Overview of 5G URLLC System and Security Aspects in 3GPP. In 2019 IEEE Conference on Standards for Communications and Networking (CSCN) (pp. 1-5). IEEE.

[11] Park, J., Samarakoon, S., Shiri, H., Abdel-Aziz, M. K., Nishio, T., Elgabli, A., \& Bennis, M. (2020). Extreme URLLC: Vision, Challenges, and Key Enablers. arXiv preprint arXiv:2001.09683.

[12] Chang, B., Zhao, G., Chen, Z., Li, P., \& Li, L. (2019, December). D2D Transmission Scheme in URLLC Enabled Real-Time Wireless Control Systems for Tactile Internet. In 2019 IEEE Global Communications Conference (GLOBECOM) (pp. 1-6). IEEE.

[13] Baratè, A., Haus, G., Ludovico, L. A., Pagani, E., \& Scarabottolo, N. (2019). 5G Technology for Augmented and Virtual Reality in Education. In Proceedings of the International Conference on Education and New Developments 2019 (END 2019) (pp. 512-516).

[14] Anand, A., De Veciana, G., \& Shakkottai, S. (2020). Joint scheduling of URLLC and eMBB traffic in $5 \mathrm{G}$ wireless networks. IEEE/ACM Transactions on Networking.

[15] Alsenwi, M., Tran, N. H., Bennis, M., Bairagi, A. K., \& Hong, C. S. (2019). eMBB-URLLC resource slicing: A risk-sensitive approach. IEEE Communications Letters, 23(4), 740-743.

[16] Pan, C., Ren, H., Deng, Y., Elkashlan, M., \& Nallanathan, A. (2019). Joint blocklength and location optimization for URLLC-enabled UAV relay systems. IEEE Communications Letters, 23(3), 498501.

[17] Singh, B., Tirkkonen, O., Li, Z., \& Uusitalo, M. A. (2017). Contention-based access for ultra-reliable low latency uplink transmissions. IEEE Wireless Communications Letters, 7(2), 182-185.

[18] Choi, Y. J., Park, S., \& Bahk, S. (2006). Multichannel random access in OFDMA wireless networks. IEEE Journal on Selected Areas in Communications, 24(3), 603-613.

[19] Sen, S., Dorsey, D. J., Guérin, R., \& Chiang, M. (2012, October). Analysis of slotted ALOHA with multipacket messages in clustered surveillance networks. In MILCOM 2012-2012 IEEE Military Communications Conference (pp. 1-6). IEEE. 